\title{
World Congress of Physical Societies
}

The role of physics and its relations with industry featured strongly during discussions at the 2nd International Conference on Research and Communications in Physics (RACIP-2) which went under the title The World Congress of Physical Societies.

The Presidents of some 50 physical societies were either present or represented at the 2nd International Conference on Research and Communications in Physics (RACIP-2) in Tokyo. Apart from the dissemination of an enormous amount of information concerning the development of physics in different parts of the world, several specific topics were at the centre of discussions. They were treated in plenary sessions and in panel discussions, and a Declaration (see insert), accepted by all the Presidents, was adopted at the end of the conference.

Various themes were considered (e.g., communication technologies in physics, notably electronic publishing; physics in developing countries; the importance of secondary school physics education) but it was the relationship of physics to society and to industry which generated the most interest. Several speakers referred to the fairly common remark that with the end of the cold war, physics must find a new role and define a new relation to society. In addition to creating knowledge, and thus being part of human culture, its contribution to the development of applied technologies has to be strengthened and better explained to politicians and the public. New paradigms are needed to place the relationship between physics and society on a new basis. It was agreed that one of the difficulties in describing the role of physics to the public is the absence of a physics industry, in contrast to a chemical industry, for example. It was proposed that the term "industrial physics" should be introduced as one step towards improving the situation. But such a term might create additional problems by distinguishing applied and industrial physics from fundamental physics. The term "physics-based industry" that has been introduced by the UK's Institute of Physics is probably more appropriate.

Regarding the relationship between physics and industry, representatives of European companies pushed for more applied research, with one representative even going so far as to say that basic research is now unnecessary. The American Physical Society (APS) wanted to do much more to integrate industrial physicists and even their employers in its activities (in the discussion it was thought that involving the latter went too far). The Japanese government's view was totally different. It does not expect much from fundamental physics in the area of new product development, but recognizes
The Pohang Accelerator Laboratory, Pohang, Korea, nearing completion in 1993. The main circular building houses a $2 \mathrm{GeV}$ synchrotron light source.

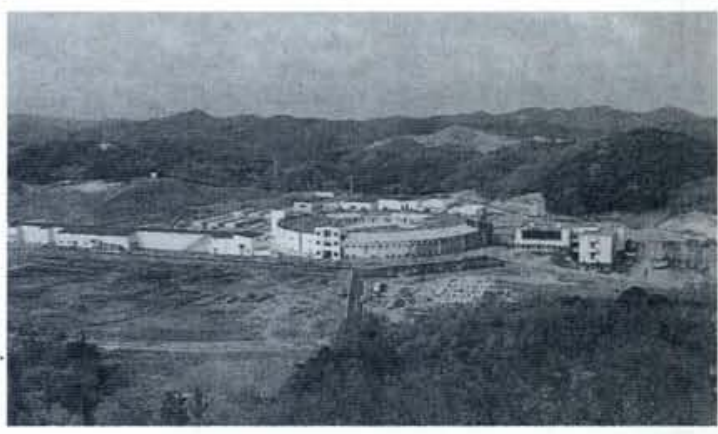

that present technologies have achieved a certain maturity and cannot be further exploited to a large extent. So the government is supporting "innovative research" in the hope that new paradigms and perspectives will come from universities. It seems that by being very short-sighted, the West risks to end up lagging behind.

Particularly impressive were reports about the economic development of South Korea where the GNP per capita has increased

100-fold from 1962 to 1994 . The country has managed to dominate some markets within a few years (e.g., shipbuilding, readonly memory chips) and to become an important competitor in others (second in motor cars and in liquid-crystal displays, both of which were invented in Europe). This was possible not only because of low salaries but also because much was invested in research (first in governmentsector institutions and later in industry). A

\section{The Tokyo Declaration of Physical Societies}

\section{Second international Conference on Research and Communications in Physics} 18-22 September 1995; Tokyo, Japan

The international physics community will continue to explore the furthest frontiers of Nature and work for the benefit of humankind. We are therefore committed to basic science as one of the highest expressions of human culture, and to applicable science as an essential instrument for human progress.

We acknowledge our obligation to contribute to the prosperity and welfare of all peoples, to dispel the ignorance which imprisons humankind in superstition, conflict and error, and to work for international amity and peace.

In pursuit of these goals,

- We shall seek to apply the physical sciences to the problems of society such as energy, the environment and renewable resources. Partnerships among scientists from the developing and developed nations will work together to improve economic conditions in all regions. We acknowledge that unless everyone shares in the sustainable growth of the international economy, no nation can be secure in its prosperity. We seek to accelerate industrial development and to ally fundamental and applied sciences with the engineering sciences in common cause. We will forge collaborations with industry to support the exploration of new and exciting scientific and technological innovations.

- We shall strive to invigorate education in the physical sciences at all levels; for children, university students and graduates, and for the wider public. Only an educated public will be prepared to benefit from the emerging technologies. And only an informed public is equipped to decide upon and support the investments needed to improve and expand the economic base of society.

- Because science is an international enterprise, dependent upon the collective intelligence of the international science community, we shall work to broaden the use of the new telecommunications technologies throughout the world. The physics community will exploit these new tools to improve inter-personal contact and dissemination of knowledge through electronic publishing, communication, research, and education.

We call upon all governments, non-governmental organizations, international agencies, sister disciplines, regional and international foundations, the international scientific unions, national academies, and others to join with us in common cause.

We shall convene the Third International Conference on Research and Communications in Physics to asses our progress in achieving these goals and to establish new objectives for the coming century. 


\section{RACIP-2}

The 2nd International Conference on Research and Communications in Physics (the first was held nine years ago in the US) took place at the United Nations University in Tokyo on 18-22 September 1995. It was organized by the Physical Society of Japan and the Japan Society of Applied Physics in coordination with the American Physical Society, the EPS, the Asia-Pacific Association of Physical Societies, and the United Nations University. H. Schopper, the EPS President, gave a talk in the opening session entitled Perspectives Towards the 21st Century (available at http:// epswww. wpfl.ch on EurophysNet under EPS Presentations) while N. Kroó, the EPS VicePresident spoke on east-west activities and led a panel discussion on east-west cooperation. new synchrotron radiation which recently came into operation testifies to the county's commitment to the importance of basic research. North Korea is also interested in joining the ITER fusion project.

One was impressed by the extent to which physicists from the US have managed to build up activities in all of the areas which were discussed. This is partly because they have a large national physical society. Europe in contrast is split and cannot yet speak with one voice. It became clear that

\title{
PHYSICS FOR DEVELOPMENT
}

\section{Helping to Reach a Minimum Level}

\begin{abstract}
Annick Suzor-Weiner, Professor of Chemical Physics at the University of Paris VI, who chairs the EPS Interdivisional Group of Physics for Development describes the Group's work which now favours joint initiatives.
\end{abstract}

The European Physical Society has always felt that there is a strong mutual interest in scientific collaboration between the more industrialised countries of northern Europe and the less advanced ones inside as well as outside Europe. This attitude was crystallised in March 1985 by launching the Interdivisional Group of Physics for Development (IGPD) chaired by Endre Lillethun from Bergen. The IGPD's 10th anniversary is an appropriate opportunity for reviewing its scope, past achievements and future perspectives.

\section{Focusing on Education and Research}

Some 220 members of EPS and national physical societies are presently IGPD members. As for all EPS Divisions and Groups, they joined by contacting the EPS Secretariat or their national society. Other individuals may be invited to participate in specific activities. A Board of 10 , including the Chairman and the Secretary, is elected by the Group's members for no longer than six years, with at least half of the Board being renewed every three years. The Board, to which a few co-opted members may be added, has overall responsibility for the Group's activities and reports to the EPS Council. It was chaired until earlier this year by Lennart Hasselgren from Uppsala who took over from Endre Lillethun in 1992.

The IGPD's overall goal is to strengthen physics and physicsrelated research and education in developing countries, i.e., countries which have not yet reached the same level of technical and industrial development as northern Europe and North America. This obviously covers a broad range of situations each calling for a different approach.

Most colleagues in northern Europe are easily convinced of

the mutual benefits stemming from collaboration with east and central Europe and southern Europe, whose well-trained young physicists may contribute much. Links with several countries in Asia and South America undergoing rapid development are also sought. However, physicists often question the value of scientific cooperation with some countries, such as many in central Africa, where the general situation is so bad that other aspects seem much more urgent. The IGPD believes that any country needs a minimum level of scientific potential and education to avoid complete technological dependence, to relate imported technologies to local resources, and to be able to grasp any opportunity for improving living conditions. It aims to help create links between universities, institutions and individuals that enhance this indispensable potential.

Whatever the level of development, the Group focuses on collaboration between groups in developing countries from the same region, and on long-term collaborative research under the joint responsibility of physicists from both developing and more industrialised countries. To help initiate projects, the IGPD promotes the organization of meetings, preferably workshops and summer schools held in developing

A modern, large-scale solar energy plant, in southern USA.

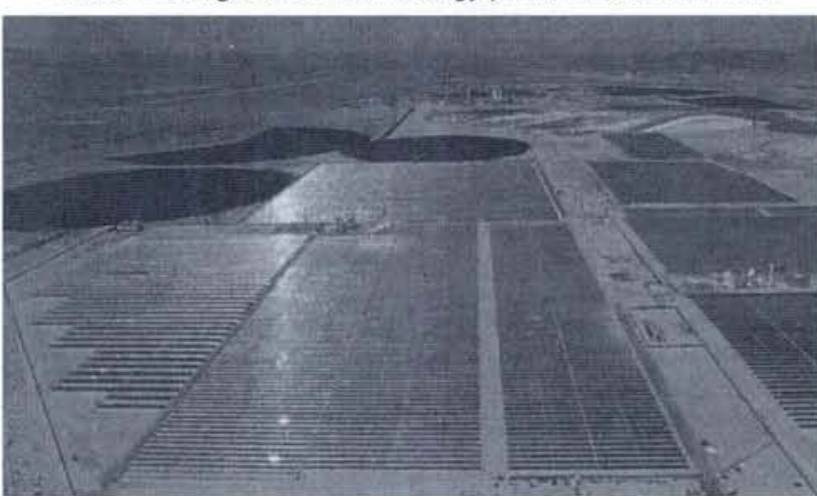

the EPS has to be strengthened if Europe wants to make a comparable contribution towards tackling the difficult issues that physics faces at the world-wide level.

The conference organizers agreed that a third event would be useful. Since the APS will be celebrating its 100 th anniversary in 1999 with a series of major events, it was decided that RACIP-3 should be in 2000 in Europe.

H. Schopper, EPS President

countries. As it has few resources for cooperation, the IGPD's possibilities are of course modest. But we can offer effective input towards developing long-term collaboration and training. Contacts with organizations supporting physics in developing countries (e.g., Third-World Academy of Sciences, International Program in the Physical Sciences, International Atomic Energy Agency, UNESCO) are essential to ensure complementarity and efficiency.

\section{A Range of Activities}

The IGPD has managed several very successful activities and some new initiatives are underway.

- Workshop on solar energy: One of the first, and most fruitful, activities of IGPD was to organize a workshop entitled Planning of Network Projects in Materials Science and Solar Energy in Nairobi, Kenya, in 1988. There were 52 participants from 18 African countries (from both English- and Frenchspeaking regions which was unusual) as well as 14 specialists from Europe. Besides the strong interest in exchanging scientific information, a concrete outcome were plans for new or enhanced cooperation projects (e.g., a collaboration between Senegal, Egypt, Thailand, Germany, and France on the study of special solar equipment in different types on southern climates, and another between Tanzania, Ethiopia, Kenya, Sudan, Uganda, Zambia, and Sweden in the field of photovoltaic cells).

- Southern European School of Physics: Southern Europe has few training schools because the institutional infrastructure that offers and promotes courses is lacking. Initiated by Henri van Regemorter (Meudon Observatory) and Siegbert Raither (UNESCO, Paris), the Southern European Schools of Physics aim at providing supplementary training for talented Ph.D. physics students and young post-doctoral physicists from Europe, through interaction with senior scientists and between young physicists from different countries. Each is hosted by a different institution whose main research field matches the topic of the school. Funding was to be provided by UNESCO, the European Commission and the Council of 